Int. J. Contemp. Math. Sci., Vol. 2, 2007, no. 16, 759 - 772

\title{
Riemann-Stieltjes Operators between Weighted Bloch and Weighted Bergman Spaces
}

\author{
Ajay K. Sharma ${ }^{1}$ \\ School of Applied Physics and Mathematics \\ Shri Mata Vaishno Devi University \\ P/O Kakryal, Udhampur-182121, India \\ aksju_76@yahoo.com \\ Som Datt Sharma \\ Department of Mathematics \\ University of Jammu, Jammu-180006, India \\ somdatt_jammu@yahoo.co.in
}

\begin{abstract}
In this paper, Riemann-Stieltjes operators between weighted Bloch and weighted Bergman spaces are considered. We characterize boundedness and compactness of these operators using certain growth properties of holomorphic symbols.
\end{abstract}

Keywords: weighted Bergman spaces, weighted Bloch spaces, RiemannStieltjes operator, Carleson measure

\section{Introduction}

Let $\mathbb{D}$ be the open unit disk in the complex plane $\mathbb{C}$. Let $g: \mathbb{D} \rightarrow \mathbb{C}$ be a holomorphic map. Denote by $H(\mathbb{D})$ the space of holomorphic functions on $\mathbb{D}$. For $f \in H(\mathbb{D})$, the Riemann-Stieltjes operator induced by $g$ is defined by

$$
T_{g} f(z)=\int_{0}^{z} f(\zeta) d g(\zeta)=\int_{0}^{1} f(t z) z g^{\prime}(t z) d t, \quad z \in \mathbb{D} .
$$

The Riemann-Stieltjes operator can be viewed as a generalization of Cesaro operator defined by

$$
T f(z)=\frac{1}{z} \int_{0}^{z} \frac{f(w)}{1-w} d(w), \quad z \in \mathbb{D} .
$$

\footnotetext{
${ }^{1}$ Supported by CSIR-grant ( F.No. 9/100(100)2002 EMR-1).
} 
Ch. Pommerenke [7] initiated the study of Riemann-Stieltjes operator on $H^{2}$, where he showed that $T_{g}$ is bounded on $H^{2}$ if and only if $g$ is in BMOA. This was extended to other Hardy spaces $H^{p}, 1 \leqslant p<\infty$, in [1] and [2] where compactness of $T_{g}$ on $H^{p}$ and Schatten class membership of $T_{g}$ on $H^{2}$, was also completely characterized in terms of the symboy $g$. Similar questions on weighted Bergman spaces were considered by A. Aleman and A. G. Siskakis in $[3]$.

Recently, several authors have studied Riemann-Stieltjes operators on different spaces of analytic functions. For example, one can refer to $([\mathbf{5}][\mathbf{8}]$ $[\mathbf{9}][\mathbf{1 0}][\mathbf{1 1}]$ and $[\mathbf{1 2}])$ for the study of these operators on Bergman spaces, Dirichlet spaces, BMOA and VMOA and related references therein. In this paper we characterize boundedness and compactness of Riemann-Stieltjes operators between between weighted Bloch and weighted Bergman spaces.

\section{Preliminaries}

In this section we review the basic concepts of weighted Bergman spaces $A_{\alpha}^{p}$ and weighted Bloch spaces $\mathcal{B}^{\alpha}$ and collect some essential facts that will be needed throughout the paper.

2.1. Weighted Bergman Spaces. Let $d A(z)$ be the area measure on $\mathbb{D}$ normalized so that area of $\mathbb{D}$ is 1 . For each $\alpha \in(-1, \infty)$, we set $d \nu_{\alpha}(z)=$ $(\alpha+1)\left(1-|z|^{2}\right)^{\alpha} d A(z), z \in \mathbb{D}$. Then $d \nu_{\alpha}$ is a probability measure on $\mathbb{D}$. For $0<p<\infty$ the weighted Bergman space $A_{\alpha}^{p}$ is defined as

$$
A_{\alpha}^{p}=\left\{f \in H(\mathbb{D}):\|f\|_{A_{\alpha}^{p}}=\left(\int_{\mathbb{D}}|f(z)|^{p} d \nu_{\alpha}(z)\right)^{1 / p}<\infty\right\} .
$$

Note that $\|f\|_{A_{\alpha}^{p}}$ is a true norm only if $1 \leqslant p<\infty$ and in this case $A_{\alpha}^{p}$ is a Banach space. For $0<p<1, A_{\alpha}^{p}$ is a non-locally convex topological vector space and $d(f, g)=\|f-g\|_{A_{\alpha}^{p}}^{p}$ is a complete metric for it. The growth of functions in the weighted Bergman spaces is essential in our study. To this end, the following sharp estimate will be useful. (see [7] p. 53.). It tells us how fast an arbitrary function from $A_{\alpha}^{p}$ grows near the boundary.

Let $f \in A_{\alpha}^{p}$. Then for every $z$ in $\mathbb{D}$, we have

$$
|f(z)| \leqslant \frac{\|f\|_{A_{\alpha}^{p}}}{\left(1-|z|^{2}\right)^{(2+\alpha) / p}}
$$

with equality holds if and only if $f$ is a constant multiple of the function

$$
k_{a}(z)=\left(\frac{1-|z|^{2}}{(1-\bar{a} z)^{2}}\right)^{(2+\alpha) / p}
$$

It can be easily shown that $\left\|k_{a}\right\|_{A_{\alpha}^{p}} \approx 1$.

2.1. Weighted Bloch Spaces. For $\alpha>0$, let $\mathcal{B}^{\alpha}$ consists of all 
analytic functions $f$ on $\mathbb{D}$ satisfying the condition

$$
\sup _{z \in \mathbb{D}}\left(1-|z|^{2}\right)^{\alpha}\left|f^{\prime}(z)\right|<\infty \text {. }
$$

Note that $\mathcal{B}^{1}=\mathcal{B}$, the usual Bloch space . For $f \in \mathcal{B}^{\alpha}$ define

$$
\|f\|_{\mathcal{B}^{\alpha}}=|f(0)|+\sup _{z \in \mathbb{D}}\left(1-|z|^{2}\right)^{\alpha}\left|f^{\prime}(z)\right|<\infty .
$$

With this norm $\mathcal{B}^{\alpha}$ is a Banach space. Integrating the estimate

$$
\left|f^{\prime}(z)\right| \leqslant \frac{\|f\|_{\mathcal{B}^{\alpha}}}{\left(1-|z|^{2}\right)^{\alpha}}
$$

we obtain

$$
|f(z)-f(0)| \leqslant \int_{0}^{1}|z|\left|f^{\prime}(t z)\right| d t \leqslant\|f\|_{\mathcal{B}^{\alpha}} \int_{0}^{1} \frac{|z|}{\left(1-t|z|^{2}\right)^{\alpha}} d t,
$$

for all $z \in \mathbb{D}$. In case $0<\alpha<1$, the integral on the right is uniformly bounded by the constant $\int_{0}^{1}(1-t)^{-\alpha} d t$, and it follows that $\mathcal{B}^{\alpha} \subset H^{\infty}$. It is easy to check that in this case the linear space $\mathcal{B}^{\alpha}$ is an algebra. In fact, Hardy and Littlewood, have shown that for $0<\alpha<1$, the space $\mathcal{B}^{\alpha}$ consist of all functions $f$ analytic on $\mathbb{D}$ satisfying the Lipschitz condition

$$
|f(z)-f(w)| \leqslant|z-w|^{1-\alpha}
$$

for all $z, w \in \mathbb{D}($ see $[4])$.

In case $1<\alpha<\infty$, the above estimate implies

$$
|f(z)-f(0)| \leqslant \frac{\|f\|_{\mathcal{B}^{\alpha}}}{\alpha-1} \frac{1}{\left(1-|z|^{2}\right)^{\alpha-1}},
$$

while for $\alpha=1$ it is well known that the following hold ([6] and $[\mathbf{1 4}])$.

$$
|f(z)-f(w)| \leqslant\|f\|_{\mathcal{B}} \beta(z, w)
$$

for $f \in \mathcal{B}$, where

$$
\beta(z, w)=\frac{1}{2} \log \frac{|1-z \bar{w}|+|z-w|}{|1-z \bar{w}|-|z-w|}
$$

is the Bergman metric on $\mathbb{D}$. From (2.4), it follows that for $f \in \mathcal{B}$,

$$
|f(z)| \leqslant \frac{1}{\log 2}\|f\|_{\mathcal{B}} \log \left(\frac{2}{1-|z|^{2}}\right) .
$$

Throughout this paper we fix some positive radius $0<r<\infty$ and consider disks $D(z, r)$ in the Bergman metric. The set

$$
D(z, r)=\{w \in \mathbb{D}: \beta(z, w)<r\}, \quad z \in \mathbb{D},
$$


is called hyprerbolic disk or Bergman disk of radius $r$ about $z$. It is well known that $D(z, r)$ is a Euclidean disk whose Euclidean center and Euclidean radius are

$$
\frac{\left(1-s^{2}\right) z}{\left(1-s^{2}|z|^{2}\right)} \quad \text { and } \quad \frac{\left(1-|z|^{2}\right) s}{\left(1-s^{2}|z|^{2}\right)}
$$

where $s=\tanh r \in(0,1)$, respectively. For fixed $r>0$. the area of $D(z, r)$ in $\mathbb{D}$ has the estimation;

$$
|D(z, r)|_{A}=\int_{D(z, r)} d A(w) \approx\left(1-|z|^{2}\right)^{2}
$$

For fixed $r>0$, it is known that if $w \in D(z, r)$, then

$$
|1-z \bar{w}| \approx\left(1-\left|z^{2}\right|\right) \quad \text { and } \quad|D(w, s)|_{A} \approx C|D(z, r)|_{A}
$$

Following lemma lists additional properties of the hyperbolic disks.

Lemma 2.2. [7] Fix $r, 0<r<\infty$. There exists a positive integer $M$ and a sequence $\left\{a_{n}\right\}$ in $\mathbb{D}$ such that :

(i) The disk $\mathbb{D}$ is covered by $\left\{D\left(a_{n}, r\right)\right\}_{n}$.

(ii) Every point in $\mathbb{D}$ belongs to at most $M$ sets in $\left\{D\left(a_{n}, 2 r\right)\right\}_{n}$.

(iii) If $n \neq m$, then $\beta\left(a_{n}, a_{m}\right) \geq \frac{r}{2}$.

We shall use these estimates in the proofs of the Theorems below. For general background of weighted Bergman spaces $A_{\alpha}^{p}$ and weighted Bloch spaces, one may consult [13] and [14] and the references therein.

\section{Riemann-Stieltjes operators from weighted Bergman spaces $A_{\alpha}^{p}$ into weighted Bloch spaces $B^{\alpha}$}

In this section we characterize boundedness and compactness of RiemannStieltjes operators from weighted Bergman spaces $A_{\alpha}^{p}$ into weighted Bloch spaces spaces $B^{\alpha}$.

The following Theorem characterizes Riemann-Stieltjes operators from weighted Bergman spaces $A_{\beta}^{p}$ into weighted Bloch spaces $\mathcal{B}^{\alpha}$.

Theorem 3.1. Let $1 \leqslant p<\infty,-1<\beta<\infty, \alpha>0$ and $g: \mathbb{D} \rightarrow \mathbb{C}$ be a holomorphic map. Then the Riemann-Stieltjes operator $T_{g}$ maps $A_{\beta}^{p}$ boundedly into $\mathcal{B}^{\alpha}$ if and only if

$$
\sup _{z \in \mathbb{D}}\left(1-|z|^{2}\right)^{(p \alpha-\beta-2) / p}\left|g^{\prime}(z)\right|<\infty .
$$


Proof. First suppose that

$$
M=\sup _{z \in \mathbb{D}}\left(1-|z|^{2}\right)^{(p \alpha-\beta-2) / p}\left|g^{\prime}(z)\right|<\infty .
$$

By (2.1), we have

$$
|f(z)| \leqslant \frac{\|f\|_{A_{\beta}^{p}}}{\left(1-|z|^{2}\right)^{(\beta+2) / p}}
$$

for all $z \in \mathbb{D}$. Thus

$$
\begin{aligned}
\left\|T_{g} f\right\|_{\mathcal{B}^{\alpha}} & =\left|T_{g} f(0)\right|+\sup _{z \in \mathbb{D}}\left(1-|z|^{2}\right)^{\alpha}\left|\left(T_{g} f\right)^{\prime}(z)\right| \\
& =\sup _{z \in \mathbb{D}}\left(1-|z|^{2}\right)^{\alpha}\left|g^{\prime}(z) f(z)\right| \\
& \leqslant \sup _{z \in \mathbb{D}}\left(1-|z|^{2}\right)^{(p \alpha-\beta-2) / p}\left|g^{\prime}(z)\right|\|f\|_{A_{\beta}^{p}} \\
& =M\|f\|_{A_{\beta}^{p}},
\end{aligned}
$$

hence $T_{g}$ maps $A_{\beta}^{p}$ boundedly into $\mathcal{B}^{\alpha}$.

Conversely, suppose $T_{g}$ maps $A_{\beta}^{p}$ boundedly into $\mathcal{B}^{\alpha}$. Fix a point $a$ in $\mathbb{D}$ and consider the function

$$
f_{a}(z)=\left(\frac{1-|a|^{2}}{(1-\bar{a} z)^{2}}\right)^{(\beta+2) / p} .
$$

Then $f_{a}$ is a function of unit norm in $A_{\beta}^{p}$. Since $T_{g}$ maps $A_{\beta}^{p}$ boundedly into $\mathcal{B}^{\alpha}$, we can find a positive constant $C$ such that

$$
\left\|T_{g} f_{a}\right\|_{\mathcal{B}^{\alpha}} \leqslant C\left\|f_{a}\right\|_{A_{\beta}^{p}}=C,
$$

for all $a \in \mathbb{D}$, hence for each point $z \in \mathbb{D}$ we have

$$
\left(1-|z|^{2}\right)^{\alpha}\left|f_{a}(z) g^{\prime}(z)\right| \leqslant C .
$$

In particular, when $z=a$, we get

$$
\left(1-|a|^{2}\right)^{\alpha}\left(\frac{1-|a|^{2}}{\left(1-|a|^{2}\right)^{2}}\right)^{(\beta+2) / p}\left|g^{\prime}(a)\right| \leqslant C,
$$

whence

$$
\left(1-|a|^{2}\right)^{(p \alpha-\beta-2) / p}\left|g^{\prime}(a)\right|<C .
$$

Since $a \in \mathbb{D}$ is arbitrary, the result follows.

Theorem 3.2. Let $1 \leqslant p<\infty,-1<\beta<\infty, \alpha>0$ and $g: \mathbb{D} \rightarrow \mathbb{C}$ be a holomorphic map. Suppose that $T_{g}$ maps $A_{\beta}^{p}$ boundedly into $\mathcal{B}^{\alpha}$. Then $T_{g}$ maps $A_{\beta}^{p}$ compactly into $\mathcal{B}^{\alpha}$ if and only if

$$
\lim _{|z| \rightarrow 1^{-}}\left(1-|z|^{2}\right)^{(p \alpha-\beta-2) / p}\left|g^{\prime}(z)\right|=0 .
$$


Proof . First suppose that (3.2) holds. Let $\left\{f_{n}\right\}$ be a bounded sequence in $A_{\beta}^{p}$ that converges to zero uniformly on compact subsets of $\mathbb{D}$. Let $M=$ $\sup _{n}\left\|f_{n}\right\|_{A_{\beta}^{p}}<\infty$. Given $\varepsilon>0$, there exist an $r \in(0,1)$ such that if $|z|>r$, then

$$
\left(1-|z|^{2}\right)^{(p \alpha-\beta-2) / p}\left|g^{\prime}(z)\right|<\varepsilon .
$$

Thus for $z \in \mathbb{D}$ such that $|z|>r$, by $(2.1)$ we have

$$
\begin{aligned}
\left(1-|z|^{2}\right)^{\alpha}\left|\left(T_{g} f_{n}\right)^{\prime}(z)\right| & =\left(1-|z|^{2}\right)^{\alpha}\left|g^{\prime}(z)\right|\left|f_{n}(z)\right| \\
& \leqslant\left(1-|z|^{2}\right)^{\alpha-(\beta-2) / p}\left|g^{\prime}(z)\right|\left\|f_{n}\right\| A_{\beta}^{p} \\
& <\varepsilon M,
\end{aligned}
$$

for all $n$. On the other hand, since $f_{n} \rightarrow 0$ uniformly on compact subsets of $\mathbb{D}$, there exist an $n_{0}$ such that if $|z| \leqslant r$ and $n \geq n_{0}$, then $\left|f_{n}^{\prime}(z)\right|<\varepsilon$. By Theorem 3.1, we have $g \in \mathcal{B}^{\alpha}$ and so we have

$$
N=\sup _{z \in \mathbb{D}}\left(1-|z|^{2}\right)^{\alpha}\left|g^{\prime}(z)\right|<\infty
$$

hence

$$
\begin{aligned}
\sup _{|z| \leqslant r}\left(1-|z|^{2}\right)^{\alpha}\left|\left(T_{g} f_{n}\right)^{\prime}(z)\right| & =\sup _{|z| \leqslant r}\left(1-|z|^{2}\right)^{\alpha}\left|g^{\prime}(z)\right| f_{n}(z) \mid \\
& <\varepsilon N .
\end{aligned}
$$

The above arguments together yield

$$
\begin{aligned}
\left\|T_{g} f_{n}\right\|_{\mathcal{B}^{\alpha}} & =\left|T_{g} f(0)\right|+\sup _{z \in \mathbb{D}}\left(1-|z|^{2}\right)^{\alpha}\left|\left(T_{g}\right)^{\prime} f_{n}(z)\right| \\
& \leqslant \sup _{|z| \leqslant r}\left(1-|z|^{2}\right)^{\alpha}\left|\left(T_{g} f_{n}\right)^{\prime}(z)\right|+\sup _{|z|>r}\left(1-|z|^{2}\right)^{\alpha}\left|\left(T_{g} f_{n}\right)^{\prime}(z)\right| \\
& \leqslant(N+M) \varepsilon .
\end{aligned}
$$

Thus

$$
\left\|T_{g} f_{n}\right\|_{\mathcal{B}^{\alpha}} \rightarrow 0 \text { as } n \rightarrow \infty
$$

hence $T_{g}$ maps $A_{\beta}^{p}$ compactly into $\mathcal{B}^{\alpha}$.

Conversely, suppose $T_{g}$ maps $A_{\beta}^{p}$ compactly into $\mathcal{B}^{\alpha}$ and (3.2) does not hold. Then there exists a positive number $\delta$ and a sequence $\left\{z_{n}\right\}$ in $\mathbb{D}$ such that $\left|z_{n}\right| \rightarrow 1$ and

$$
\left(1-\left|z_{n}\right|^{2}\right)^{(p \alpha-\beta-2) / p}\left|g^{\prime}\left(z_{n}\right)\right| \geq \delta,
$$

for all $n$. For each $n$, consider the function $f_{n}$ defined as

$$
f_{n}(z)=\left(\frac{1-\left|z_{n}\right|^{2}}{\left(1-\bar{z}_{n} z\right)^{2}}\right)^{(\beta+2) / p}, \quad z \in \mathbb{D} .
$$


Then the sequence $\left\{f_{n}\right\}$ is norm bounded and $f_{n} \rightarrow 0$ uniformly on compact subsets of $\mathbb{D}$, it follows that a subsequence of $\left\{T_{g} f_{n}\right\}$ tends to 0 in $\mathcal{B}^{\alpha}$. On the other hand,

$$
\begin{aligned}
|| T_{g} f_{n} \|_{\mathcal{B}^{\alpha}} \geq\left(1-\left|z_{n}\right|^{2}\right)^{\alpha}\left|\left(T_{g} f_{n}\right)^{\prime}\left(z_{n}\right)\right| \\
\quad=\left(1-\left|z_{n}\right|^{2}\right)^{\alpha}\left|g^{\prime}\left(z_{n}\right)\right|\left|f_{n}\left(z_{n}\right)\right| \\
\quad=\left(1-\left|z_{n}\right|^{2}\right)^{(p \alpha-\beta-2) / p}\left|g^{\prime}\left(z_{n}\right)\right| \\
\geq \delta
\end{aligned}
$$

which is absurd. Hence we are done.

\section{Riemann-Stieltjes operators between weighted Bloch spaces $B^{\alpha}$}

In this section we characterize boundedness and compactness of RiemannStieltjes operators between weighted Bloch spaces $B^{\alpha}$.

Theorem 4.1. Let $\alpha>0, \beta>0$ and $g: \mathbb{D} \rightarrow \mathbb{C}$ be a holomorphic map.

(i) If $0<\alpha<1$, then $T_{g}$ maps $\mathcal{B}^{\alpha}$ boundedly into $\mathcal{B}^{\beta}$ if and only if $g \in \mathcal{B}^{\beta}$.

(ii) Operator $T_{g}$ maps $\mathcal{B}$ boundedly into $\mathcal{B}^{\beta}$ if and only if

$$
\sup _{z \in \mathbb{D}}\left(1-|z|^{2}\right)^{\beta}\left|g^{\prime}(z)\right| \log \frac{2}{\left(1-|z|^{2}\right)}<\infty .
$$

(iii) If $\alpha>1$, then $T_{g}$ maps $\mathcal{B}^{\alpha}$ boundedly into $\mathcal{B}^{\beta}$ if and only if

$$
\sup _{z \in \mathbb{D}}\left(1-|z|^{2}\right)^{1+\beta-\alpha}\left|g^{\prime}(z)\right|<\infty
$$

Proof. First we consider the case $\alpha>1$. Suppose $T_{g}$ maps $\mathcal{B}^{\alpha}$ boundedly into $\mathcal{B}^{\beta}$. Consider the function

$$
f_{a}(z)=\frac{1-|a|^{2}}{(1-\bar{a} z)^{\alpha}}, \quad z \in \mathbb{D} .
$$

Then $\left\|f_{a}\right\|_{\mathcal{B}^{\beta}} \leqslant 1+2 \alpha$. Thus $f_{a} \in \mathcal{B}^{\alpha}$ and $M=\sup \left\{\left\|f_{a}\right\|_{\mathcal{B}^{\beta}}: a \in \mathbb{D}\right\} \leqslant 1+2 \alpha$. Since $T_{g}$ maps $\mathcal{B}^{\alpha}$ boundedly into $\mathcal{B}^{\beta}$, we can find a positive constant $C$ such that

$$
\left\|T_{g} f_{a}\right\|_{\mathcal{B}^{\beta}} \leqslant C\left\|f_{a}\right\|_{\mathcal{B}^{\alpha}} \leqslant C M
$$

for each $a \in \mathbb{D}$, hence for each $z \in \mathbb{D}$, we have

$$
\begin{aligned}
\left(1-|z|^{2}\right)^{\beta}\left|g^{\prime}(z)\right|\left|f_{a}(z)\right| & =\left(1-|z|^{2}\right)^{\beta}\left|\left(T_{g} f_{a}\right)^{\prime}(z)\right| \\
& \leqslant C M .
\end{aligned}
$$


In particular, when $z=a$, we have

$$
\left(1-|a|^{2}\right)^{1+\beta-\alpha}\left|g^{\prime}(a)\right| \leqslant C M .
$$

Since $a \in \mathbb{D}$ is arbitrary, the result follows.

Conversely, suppose that

$$
M=\sup _{z \in \mathbb{D}}\left(1-|z|^{2}\right)^{1+\beta-\alpha}\left|g^{\prime}(z)\right|<\infty
$$

By (2.3), we have

$$
|f(z)-f(0)| \leqslant \frac{\|f\|_{\mathcal{B}^{\alpha}}}{(\alpha-1)\left(1-|z|^{2}\right)^{(\alpha-1)}}
$$

for all $z \in \mathbb{D}$, independent of $f \in \mathcal{B}^{\alpha}$. Since

$$
\begin{aligned}
\left\|T_{g} f\right\|_{\mathcal{B}^{\beta}} & =\left|T_{g} f(0)\right|+\sup _{z \in \mathbb{D}}\left(1-|z|^{2}\right)^{\beta}\left|\left(T_{g} f\right)^{\prime}(z)\right| \\
& \leqslant \sup _{z \in \mathbb{D}}\left(1-|z|^{2}\right)^{\beta}|f(z)-f(0)|\left|g^{\prime}(z)\right|+|f(0)| \sup _{z \in \mathbb{D}}\left(1-|z|^{2}\right)^{\beta}\left|g^{\prime}(z)\right| \\
& \leqslant \sup _{z \in \mathbb{D}}\left(1-|z|^{2}\right)^{\beta}\left|g^{\prime}(z)\right| \frac{\|f\|_{\mathcal{B}^{\beta}}}{(\alpha-1)\left(1-|z|^{2}\right)^{(\alpha-1)}}+C \sup _{z \in \mathbb{D}}\left(1-|z|^{2}\right)^{\beta}\left|g^{\prime}(z)\right| \\
& \leqslant\left(C M+\frac{M}{(\alpha-1)}\right)|| f \|_{\mathcal{B}^{\beta} .}
\end{aligned}
$$

Hence $T_{g}$ maps $\mathcal{B}^{\alpha}$ boundedly into $\mathcal{B}^{\beta}$. This completes the proof of (iii). Next, we will prove $(i i)$. Suppose $T_{g}$ maps $\mathcal{B}$ boundedly into $\mathcal{B}^{\beta}$. For $a \in \mathbb{D}$, let

$$
f_{a}(z)=\log \frac{2}{(1-\bar{a} z)}, \quad z \in \mathbb{D} .
$$

Then $f_{a} \in \mathcal{B}$ and $\left\|f_{a}\right\|_{\mathcal{B}} \leqslant 3$. So

$$
\begin{aligned}
3\left\|T_{g}\right\|_{\mathcal{B}^{\beta}} & \geq\left\|T_{g} f_{a}\right\|_{\mathcal{B}^{\beta}} \\
& =\left|T_{g} f_{a}(0)\right|+\sup _{z \in \mathbb{D}}\left(1-|z|^{2}\right)^{\beta}\left|\left(T_{g} f_{a}\right)^{\prime}(z)\right| \\
& \geq\left(1-|a|^{2}\right)^{\beta}\left|g^{\prime}(a)\right|\left|f_{a}(a)\right| \\
& =\left(1-|a|^{2}\right)^{\beta}\left|g^{\prime}(a)\right| \log \frac{2}{\left(1-|a|^{2}\right)} .
\end{aligned}
$$

Since $a \in \mathbb{D}$ is arbitrary, the result follows.

Convesely, suppose that

$$
M=\sup _{z \in \mathbb{D}}\left(1-|z|^{2}\right)^{\beta}\left|g^{\prime}(z)\right| \log \frac{2}{\left(1-|z|^{2}\right)}<\infty .
$$


By $(2.5)$, for $f \in \mathcal{B}^{\alpha}$, we have

$$
\begin{aligned}
\left\|T_{g} f\right\|_{\mathcal{B}^{\beta}} & =\left|T_{g} f(0)\right|+\sup _{z \in \mathbb{D}}\left(1-|z|^{2}\right)^{\beta}\left|\left(T_{g} f\right)^{\prime}(z)\right| \\
& \leqslant \sup _{z \in \mathbb{D}}\left(1-|z|^{2}\right)^{\beta}\left|g^{\prime}(z)\right| \frac{1}{\log 2} \log \frac{2}{\left(1-|z|^{2}\right)}\|f\|_{\mathcal{B}^{\alpha}} \\
& =\frac{1}{\log 2} M\|f\|_{\mathcal{B}},
\end{aligned}
$$

hence $T_{g}$ maps $\mathcal{B}$ boundedly into $\mathcal{B}^{\beta}$. This completes the proof of (ii). Finally we will prove $(i)$. First suppose that $T_{g}$ maps $\mathcal{B}^{\alpha}$ boundedly into $\mathcal{B}^{\beta}$, then

$$
g=g(0)+T_{g} 1 \in \mathcal{B}^{\beta} .
$$

Conversely, suppose that $g \in \mathcal{B}^{\beta}$. Then

$$
|f(z)| \leqslant\|f\|_{\mathcal{B}^{\alpha}}\left(1+\left(1-|z|^{2}\right)^{1-\alpha}\right), \quad \alpha \neq 1, z \in \mathbb{D} .
$$

Thus, if $f \in \mathcal{B}^{\alpha}$, then

$$
\begin{aligned}
\left\|T_{g} f\right\|_{\mathcal{B}^{\beta}} & \leqslant \sup _{z \in \mathbb{D}}\left(1-|z|^{2}\right)^{\beta}\left|\left(T_{g} f\right)^{\prime}(z)\right| \\
& \leqslant \sup _{z \in \mathbb{D}}\left(\left(1-|z|^{2}\right)^{\beta}+\left(1-|z|^{2}\right)^{\beta+1-\alpha}\right)\left|g^{\prime}(z)\right|\|f\|_{\mathcal{B}^{\alpha}} \\
& \leqslant C \sup _{z \in \mathbb{D}}\left(\left(1-|z|^{2}\right)^{\beta}\right)\left|g^{\prime}(z)\right|\|f\|_{\mathcal{B}^{\alpha}} \\
& \leqslant C\|g\|_{\mathcal{B}^{\beta}}\|f\|_{\mathcal{B}^{\alpha}} .
\end{aligned}
$$

As a result, $T_{g}$ maps $\mathcal{B}^{\alpha}$ boundedly into $\mathcal{B}^{\beta}$.

Lemma 4.2.[6] Let $0<\alpha<1$ and let $T$ be a bounded linear operator from $\mathcal{B}^{\alpha}$ into a normed linear space $X$. Then $T$ is compact if and only if $\left\|T f_{n}\right\|_{X} \rightarrow 0$, whenever $\left\{f_{n}\right\}$ is a bounded sequence in $\mathcal{B}^{\alpha}$ that converges to zero uniformly on $\overline{\mathbb{D}}$.

Theorem 4.3. Let $\alpha>0, \beta>0$ and $g: \mathbb{D} \rightarrow \mathbb{C}$ be a holomorphic map. Suppose that $T_{g}$ maps $\mathcal{B}^{\alpha}$ boundedly into $\mathcal{B}^{\beta}$.

(i) If $0<\alpha<1$, then $T_{g}$ maps $\mathcal{B}^{\alpha}$ compactly into $\mathcal{B}^{\beta}$.

(ii) Operator $T_{g}$ maps $\mathcal{B}$ compactly into $\mathcal{B}^{\beta}$ if and only if

$$
\lim _{|z| \rightarrow 1}\left(1-|z|^{2}\right)^{\beta}\left|g^{\prime}(z)\right| \log \frac{2}{\left(1-|z|^{2}\right)}=0 .
$$

(iii) If $\alpha>1$, then $T_{g}$ maps $\mathcal{B}^{\alpha}$ compactly into $\mathcal{B}^{\beta}$ if and only if

$$
\lim _{|z| \rightarrow 1}\left(1-|z|^{2}\right)^{1+\beta-\alpha}\left|g^{\prime}(z)\right|=0 .
$$


Proof. First we consider the case $\alpha>1$. To prove that the condition in (iii) is sufficient for compactness of the operator $T_{g}$ from $\mathcal{B}^{\alpha}$ into $\mathcal{B}^{\beta}$, it is enough to show that if $\left\{f_{n}\right\}$ is a bounded sequence in $\mathcal{B}^{\alpha}$ that converges to zero uniformly on compact subsets of $\mathbb{D}$, then $\lim _{n \rightarrow \infty}\left\|T_{g} f_{n}\right\|_{\mathcal{B}^{\beta}}=0$. Let $M=\sup _{n}\left\|f_{n}\right\|_{\mathcal{B}^{\alpha}}<$ $\infty$. Given $\varepsilon>0$, there exists an $r \in(0,1)$ such that, if $|z|>r$, then

$$
\left(1-|z|^{2}\right)^{1+\beta-\alpha}\left|g^{\prime}(z)\right|<\varepsilon \text {. }
$$

By (2.3), we have

$$
\left|f_{n}(z)-f_{n}(0)\right| \leqslant \frac{|| f_{n} \|_{\mathcal{B}^{\alpha}}}{(\alpha-1)\left(1-|z|^{2}\right)^{(\alpha-1)}}
$$

for all $z \in \mathbb{D}$. Since

$$
\begin{aligned}
\left\|T_{g} f_{n}\right\|_{\mathcal{B}^{\beta}} & =\left|T_{g} f_{n}(0)\right|+\sup _{z \in \mathbb{D}}\left(1-|z|^{2}\right)^{\beta}\left|\left(T_{g} f_{n}\right)^{\prime}(z)\right| \\
& \leqslant \sup _{z \in \mathbb{D}}\left(1-|z|^{2}\right)^{\beta}\left|f_{n}(z)-f_{n}(0)\right|\left|g^{\prime}(z)\right|+\left|f_{n}(0)\right| \sup _{z \in \mathbb{D}}\left(1-|z|^{2}\right)^{\beta}\left|g^{\prime}(z)\right| \\
& \leqslant \sup _{|z| \leqslant r}\left(1-|z|^{2}\right)^{\beta}\left|g^{\prime}(z)\right|\left|f_{n}(z)-f_{n}(0)\right|+\left|f_{n}(0)\right| \sup _{|z| \leqslant r}\left(1-|z|^{2}\right)^{\beta}\left|g^{\prime}(z)\right| \\
& +\sup _{|z|>r}\left(1-|z|^{2}\right)^{\beta}\left|g^{\prime}(z)\right|\left|f_{n}(z)-f_{n}(0)\right|+\left|f_{n}(0)\right| \sup _{|z|>r}\left(1-|z|^{2}\right)^{\beta}\left|g^{\prime}(z)\right| \\
\leqslant & \sup _{|z| \leqslant r}\left(1-|z|^{2}\right)^{\beta}\left|g^{\prime}(z)\right|\left|f_{n}(z)-f_{n}(0)\right|+\left|f_{n}(0)\right| \sup _{|z| \leqslant r}\left(1-|z|^{2}\right)^{\beta}\left|g^{\prime}(z)\right| \\
& +\sup _{|z|>r}\left(1-|z|^{2}\right)^{\beta} \frac{\left.\left|g^{\prime}(z)\right||| f\right|_{\mathcal{B}^{\alpha}}}{(\alpha-1)\left(1-|z|^{2}\right)^{(\alpha-1)}}+\left|f_{n}(0)\right| \sup _{|z|>r}\left(1-|z|^{2}\right)^{\beta}\left|g^{\prime}(z)\right| \\
& \varepsilon\left(4|| g \|_{\mathcal{B}^{\beta}}+\frac{M}{\alpha-1}+M\right) \quad \text { as } n \geq n_{0} .
\end{aligned}
$$

Thus, $T_{g}$ maps $\mathcal{B}^{\alpha}$ compactly into $\mathcal{B}^{\beta}$.

Conversely, suppose that $T_{g}$ maps $\mathcal{B}^{\alpha}$ compactly into $\mathcal{B}^{\beta}$ and (iii) does not hold. Then there exists a positive number $\delta$ and a sequence $\left\{z_{n}\right\}$ in $\mathbb{D}$ such that $\left|z_{n}\right| \rightarrow 1$ and

$$
\left(1-\left|z_{n}\right|^{2}\right)^{1+\beta-\alpha}\left|g^{\prime}\left(z_{n}\right)\right| \geq \delta
$$

for all $n$. For each $n$, let

$$
f_{n}(z)=\frac{1-\left|z_{n}\right|^{2}}{\left(1-\bar{z}_{n} z\right)^{\alpha}}, \quad z \in \mathbb{D} .
$$

Then the sequence $f_{n}$ is norm bounded and $f_{n} \rightarrow 0$ uniformly on compact subsets of $\mathbb{D}$. Hence there exists a subsequence of $\left\{T_{g} f_{n}\right\}$ which tends to 0 in $\mathcal{B}^{\beta}$. On the other hand,

$$
\begin{aligned}
\left\|T_{g} f_{n}\right\|_{\mathcal{B}^{\beta}} & \geq\left(1-\left|z_{n}\right|^{2}\right)^{\beta}\left|\left(T_{g} f_{n}\right)^{\prime}\left(z_{n}\right)\right| \\
& =\left(1-\left|z_{n}\right|^{2}\right)^{\beta}\left|g^{\prime}\left(z_{n}\right)\right|\left|f_{n}\left(z_{n}\right)\right| \\
& =\left(1-\left|z_{n}\right|^{2}\right)^{1+\beta-\alpha}\left|g^{\prime}\left(z_{n}\right)\right| \\
& \geq \delta
\end{aligned}
$$


which is absurd. Hence we are done.

Next, we will prove (ii). Let $\left\{f_{n}\right\}$ is a bounded sequence in $\mathcal{B}$ that converges to zero uniformly on compact subsets of $\mathbb{D}$. Let $M=\sup _{n}\left\|f_{n}\right\|_{\mathcal{B}}<\infty$. Given $\varepsilon>0$, there exists an $r \in(0,1)$ such that, if $|z|>r$, then

$$
\left(1-|z|^{2}\right)^{\beta}\left|g^{\prime}(z)\right| \log \frac{2}{\left(1-|z|^{2}\right)}<\varepsilon \text {. }
$$

By (2.5), we have

$$
\left|f_{n}(z)\right| \leqslant \frac{1}{\log 2}\left\|f_{n}\right\|_{\mathcal{B}} \log \frac{2}{\left(1-|z|^{2}\right)}
$$

for all $z \in \mathbb{D}, f \in \mathcal{B}^{\alpha}$. Also, by Theorem 4.1, we have $g \in \mathcal{B}^{\beta}$ and so, for the above $\varepsilon$, we can find $n_{0} \in \mathbb{N}$ such that

$$
\begin{aligned}
\left\|T_{g} f_{n}\right\|_{\mathcal{B}^{\beta}} & =\left|T_{g} f_{n}(0)\right|+\sup _{z \in \mathbb{D}}\left(1-|z|^{2}\right)^{\beta}\left|g^{\prime}(z)\right| f_{n}(z) \mid \\
& \leqslant \sup _{|z| \leqslant r}\left(1-|z|^{2}\right)^{\beta}\left|g^{\prime}(z)\right|\left|f_{n}(z)\right|+\sup _{|z|>r}\left(1-|z|^{2}\right)^{\beta}\left|g^{\prime}(z)\right|\left|f_{n}(z)\right| \\
& \leqslant \varepsilon\left(\|g\|_{\mathcal{B}^{\beta}}+M\right) \text { for } n \geq n_{0} .
\end{aligned}
$$

Thus $T_{g}$ maps $\mathcal{B}$ compactly into $\mathcal{B}^{\beta}$. Conversely, suppose $T_{g}$ maps $\mathcal{B}$ compactly into $\mathcal{B}^{\beta}$ and condition in (ii ) does not hold. Then there exists a positive number $\delta$ and a sequence $\left\{z_{n}\right\}$ in $\mathbb{D}$ such that $\left|z_{n}\right| \rightarrow 1$ and

$$
\left(1-\left|z_{n}\right|^{2}\right)^{\beta}\left|g^{\prime}\left(z_{n}\right)\right| \log \frac{2}{\left(1-\left|z_{n}\right|^{2}\right)} \geq \delta,
$$

for all $n$. For each $n$, let

$$
f_{n}(z)=\log \frac{2}{\left(1-\bar{z}_{n} z\right)}, \quad z \in \mathbb{D} .
$$

Then the sequence $f_{n}$ is norm bounded and $f_{n} \rightarrow 0$ uniformly on compact subsets of $\mathbb{D}$. By the compactness of $T_{g}$ we can find a subsequence of $\left\{T_{g} f_{n}\right\}$ which tends to 0 in $\mathcal{B}^{\beta}$. On the other hand,

$$
\begin{aligned}
\left\|T_{g} f_{n}\right\|_{\mathcal{B}^{\beta}} & \geq\left(1-\left|z_{n}\right|^{2}\right)^{\beta}\left|\left(T_{g} f_{n}\right)^{\prime}\left(z_{n}\right)\right| \\
& =\left(1-\left|z_{n}\right|^{2}\right)^{\beta}\left|g^{\prime}\left(z_{n}\right)\right|\left|f_{n}\left(z_{n}\right)\right| \\
& =\left(1-\left|z_{n}\right|^{2}\right)^{\beta}\left|g^{\prime}\left(z_{n}\right)\right| \log \frac{2}{\left(1-\left|z_{n}\right|^{2}\right)} \\
& \geq \delta,
\end{aligned}
$$

which is absurd. We are done.

Finally, we will prove (i). Suppose that $\sup _{n}\left\|f_{n}\right\|_{\mathcal{B}^{\alpha}} \leqslant M$ and $f_{n} \rightarrow 0$ uniformly on $\overline{\mathbb{D}}$. Then

$$
\sup _{z \in \mathbb{D}}\left(1-|z|^{2}\right)^{\beta}\left|g^{\prime}(z)\right|\left|f_{n}(z)\right| \leqslant \sup _{|z| \leqslant 1}\left|f_{n}(z)\right|\|g\|_{\mathcal{B}^{\beta}} \longrightarrow 0 \quad \text { as } n \rightarrow \infty .
$$


It follows from Lemma 4.2 that $T_{g}$ maps $\mathcal{B}^{\alpha}$ compactly into $\mathcal{B}^{\beta}$.

Lemma 4.4. Let $1 \leqslant p<q<\infty$ and $-1<\alpha<\infty$. Then the injection map from $A_{\alpha}^{q}$ into $A_{\alpha}^{p}$ is compact.

Proof Let $\left\{f_{n}\right\}_{n=1}^{\infty}$ be a bounded sequence in $A_{\alpha}^{q}$, and let $M=\sup _{n \in \mathbb{N}}\left\|f_{n}\right\|_{A_{\alpha}^{q}} \leqslant$ $\infty$. By 2.1, $\left\{f_{n}: n \in \mathbb{N}\right\}$ is a normal family, hence we can find a subsequence $\left\{f_{n_{k}}\right\}$ of $\left\{f_{n}\right\}$ that converges uniformly on compact subsets of $\mathbb{D}$ to an analytic function $f$. By Fatou's lemma,

$$
\int_{\mathbb{D}}|f(z)|^{p} \nu_{\alpha}(z) \leqslant \lim \inf _{k \rightarrow \infty} \int_{\mathbb{D}}\left|f_{n k}(z)\right|^{p} \nu_{\alpha}(z) \leqslant M^{q}<\infty .
$$

Since $p<q$, it follows that $f \in A_{\alpha}^{q}$. We claim that $\left\{f_{n_{k}}\right\}$ converges to $f$ in $A_{\alpha}^{p}$. Let $\varepsilon>0$ and let $\Omega$ be an arbitrary compact subset of $\mathbb{D}$. Now

$$
\begin{aligned}
\int_{\mathbb{D} \backslash \Omega}\left|\left(f_{n_{k}}-f\right)(z)\right|^{p} \nu_{\alpha}(z) & \leqslant\left(\int_{\mathbb{D} \backslash \Omega}\left|\left(f_{n_{k}}-f\right)(z)\right|^{q} \nu_{\alpha}(z)\right)^{p / q}\left(\int_{\mathbb{D} \backslash \Omega} \nu_{\alpha}(z)\right)^{1-p / q} \\
& \leqslant\left(\int_{\mathbb{D}}\left|\left(f_{n_{k}}-f\right)(z)\right|^{q} \nu_{\alpha}(z)\right)^{p / q}\left(\nu_{\alpha}(\mathbb{D} \backslash \Omega)\right)^{1-p / q} \\
& \leqslant\left(2^{q} \int_{\mathbb{D}} \mid\left(\left.f_{n_{k}}(z)\right|^{q}+|f(z)|^{q}\right) \nu_{\alpha}(z)\right)^{p / q}\left(\nu_{\alpha}(\mathbb{D} \backslash \Omega)\right)^{1-p / q} \\
& \leqslant 2^{p}\left(\left.\left\|\left.f_{n_{k}}\right|_{A_{\alpha}^{q}} ^{p}+\right\| f\right|_{A_{\alpha}^{q}} ^{p}\left(\nu_{\alpha}(\mathbb{D} \backslash \Omega)\right)^{1-p / q}\right. \\
& \leqslant 2^{p+1} M^{p}\left(\nu_{\alpha}(\mathbb{D} \backslash \Omega)\right)^{1-p / q},
\end{aligned}
$$

where in the first line we have used Holder's inequality and the elementary inequalities

$$
(x+y)^{a} \leqslant 2^{a}\left(x^{a}+y^{b}\right), \quad(x+y)^{b} \leqslant\left(x^{b}+y^{b}\right)
$$

which holds when $x, y \geq 0$, and $0<b<1$. By choosing the compact set $\Omega$ so that $\mathbb{D} \backslash \Omega$ has sufficiently small area, we obtain

$$
\int_{\mathbb{D} \backslash \Omega}\left|\left(f_{n_{k}}-f\right)(z)\right|^{p} \nu_{\alpha}(z)<\varepsilon / 2
$$

for $k$ large enough. On the other hand, since $f_{n} \rightarrow f$ uniformly on $\Omega$ we can choose $k$ large enough so that

$$
\int_{\Omega}\left|\left(f_{n k}-f\right)(z)\right|^{p} \nu_{\alpha}(z)<\varepsilon / 2 .
$$

Thus $\left\{f_{n_{k}}\right\}$ converges to $f$ in $A_{\alpha}^{p}$. Hence the injection map is compact.

Corollary 4.5. Let $1 \leqslant p<\infty,-1<\beta<\infty, \alpha>0$ and $g: \mathbb{D} \rightarrow \mathbb{C}$ be holomorphic. Then $T_{g}$ maps $\mathcal{B}^{\alpha}$ compactly into $A_{\beta}^{p}$ if and only if $T_{g}$ maps $\mathcal{B}^{\alpha}$ boundedly into $\mathcal{B}^{\beta}$.

Proof. Suppose $T_{g}$ maps $\mathcal{B}^{\alpha}$ boundedly into $\mathcal{B}^{\beta}$, thus also into the large space 
$A_{\beta}^{p}$. Since convergence in either space implies uniform convergence on compact sets, it follows from closed graph theorem that $T_{g}$ maps $\mathcal{B}^{\alpha}$ boundedly into $A_{\beta}^{p}$. In order to show that $T_{g}$ maps $\mathcal{B}^{\alpha}$ compactly into $A_{\beta}^{p}$, choose any $q$ such that $q>p$ and factorize $T_{g}$ through the intermediate space $A_{\beta}^{q}$ :

$$
\mathcal{B}^{\alpha} \stackrel{\widetilde{T}_{g}}{\longrightarrow} A_{\beta}^{q} \stackrel{I}{\longrightarrow} A_{\beta}^{p}
$$

where $\widetilde{T}_{g}$ is the Riemann-Stieltjes operator from $\mathcal{B}^{\alpha}$ to $A_{\beta}^{q}$, and $I$ is the injection map. Since $I$ is compact and $\widetilde{T}_{g}$ is bounded, so $T_{g}$ maps $\mathcal{B}^{\alpha}$ compactly into $A_{\beta}^{p}$.

\section{References}

[1] A. Aleman and A. G. Siskakis, 'An integral operator on $H^{p}$ and Hardy inequality', J. Anal. Math. 85 (2001), 157-176.

[2] A. Aleman and A. G. Siskakis,'An integral operator on $H^{p}$, Complex variables. 28 (1995), 149-158. 46 (1997), 337-356.

[3] A. Aleman and A. G. Siskakis, 'Integral operators on Bergman spaces', Indiana Univ. Math. J. 46 (1997), 337-356.

[4] P. L. Duren, 'Theory of $H^{p}$ spaces', Academic Press, New York, 1970.

[5] B.D. MacCluer and R. Zhao, 'Vanishing logarithmic Carleson measures', Illinois J. Math. 46 (2002), 507-518.

[6] S. Ohno, K. Stroethoff and R. Zhao, 'Weighted composition operators between Bloch-type spaces', Rocky Mountain J. Math., 33 (2003), 191215.

[7] C. Pommerenke,'Schlichte Funktionen und analytische Funktionen von beschrankter mittlerer Oszillation', Comment. Math. Helv. 52 (1977), 591-602.

[8] A. G. Siskakis and R. Zhao,'A Volterra type operator on spaces of analytic functions', Contemp. Math. 232 (1999), 299-311.

[9] J. Xiao, 'Cesaro operators on Hardy, BMOA and Bloch spaces', Arch. Math. 68 (1997), 398-406.

[10] J. Xiao,'The $\bar{\partial}$-problem for multipliers of the Sobolev space', Acta. Sci Math. (Szeged) 69 (2003), 605-618.

[11] J. Xiao, 'Riemann-Stieltjes operators on weighted Bloch and Bergman spaces of the unit ball', J. London Math. Soc. 70 (2004), 199-214. 
[12] R. Zhao, 'On logarithmic Carleson measures', Contemp. Math. 232 (1999), 299-311.

[13] K. Zhu, 'Operator theory in function spaces', Marcel Dekker, New York, 1990.

[14] K. Zhu, 'Bloch type spaces of analytic functions', Rocky Mountain J. Math., 23 (1993), 1143-1177.

Received: October 5, 2006 